\title{
Magnetic Resonance Imaging Features of Traumatic Patellofemoral Dislocation
}

\author{
Kunal Mohan ${ }^{1}$, Prasad Ellanti ${ }^{1}$, Marc Lincoln ${ }^{1}$, Tom McCarthy ${ }^{1}$ \\ 1. Orthopaedics, Saint James's Hospital, Dublin, IRL
}

Corresponding author: Kunal Mohan, kmohan@tcd.ie

\begin{abstract}
Acute traumatic patellar dislocations are encountered with relative frequency, making up 3\% of all knee injuries. Typically witnessed in younger patients following sporting injuries, this injury can be debilitating, potentially leading to recurrent dislocation, pain, reduction in activity and patellofemoral osteoarthritis.

Management of this injury remains controversial, and as such detailed magnetic resonance imaging (MRI) is increasingly recommended to help illustrate the exact nature of osteochondral and soft tissue injury, with a view to assessing the anatomical sequelae of patellar dislocation as well as the potential of recurrence and dictating the need for either conservative or surgical management in the acute setting. As such, awareness of the typical MRI findings in traumatic patellar dislocations may potentially aid in pursuing appropriate intervention for this pathology.
\end{abstract}

This case describes a 33-year-old gentleman presenting to the emergency department following patellar dislocation. After failed departmental closed reduction, this patient progressed on to definitive anatomical MRI assessment followed by acute surgical intervention in the form of medial patellofemoral ligament (MPFL) repair. This case allows for both illustration and discussion of typical radiological features associated with traumatic patellar dislocation.

Categories: Orthopedics

Keywords: acute, patella, dislocation, mri, radiographic, features, trauma, primary

\section{Introduction}

Acute traumatic patellar dislocations are encountered with relative frequency, making up 3\% of all knee injuries [1]. Typically witnessed in younger patients following sporting injuries, patellar dislocations generally occur laterally following either a twisting injury or direct impact, with resultant disruption of the medial patellofemoral ligament (MPFL), the primary restraint to lateral patellar subluxation, and accompanying retinaculum occurring in approximately $90 \%$ of affected patients [1,2]. While infrequent, this injury can be debilitating, potentially leading to recurrence in up to $40 \%$ with associated pain, reduction in activity and patellofemoral osteoarthritis [2].

Received 12/05/2018 Review began 12/06/2018 Review ended 12/12/2018 Published 12/14/2018

(c) Copyright 2018 Mohan et al. This is an open access article distributed under the terms of the Creative Commons Attribution License CC-BY 3.0., which permits unrestricted use, distribution, and reproduction in any medium, provided the original author and source are credited.
Assessment of a primary traumatic patellar dislocation is multifaceted, involving initial clinical history and examination followed by initial plain radiographic evaluation involving anteroposterior, lateral and axial views [3]. While plain radiographs are useful in confirming the diagnosis, magnetic resonance imaging (MRI) is frequently recommended for definitive radiological evaluation following acute traumatic patellar dislocation, so to further assess soft tissue pathology and osteochondral injury and dictate the need for surgical intervention $[2,3]$.

The following case report allows for illustration and discussion of some of the typical MRI findings one may encounter following acute patellar dislocation that may potentially precipitate subsequent definitive surgical intervention.

\section{Case Presentation}

A 33-year-old gentleman presented to the emergency department describing substantial right knee pain and swelling following a rotational injury. Inspection indicated significant joint effusion and subsequent examination showed absence of straight leg raise on the affected side. Plain radiographic evaluation of the knee showed lateral displacement of the patella (Figure 1) confirming the clinical suspicion of a patellofemoral dislocation. Attempted closed reduction in the emergency department was unsuccessful, with the patella irreducible due to both an appreciable mechanical block and limited patient tolerance despite sedation. An MRI was thus performed to both assess any potential obstruction to closed reduction as well as to definitively evaluate the associated soft-tissue injury prior to determining the need for subsequent surgical intervention. 


\section{Cureus}

Radiological investigations displayed evidence of lateral patellar dislocation on plain radiograph (Figure 1), as well as MRI features of traumatic patellar dislocation including patellar displacement, associated patellofemoral effusion, MPFL and medial patellar retinaculum tears and avulsed patellar cartilage (Figures 2-4). Additional MRI assessment showed a raised sulcus angle (>140) (Figure 2).

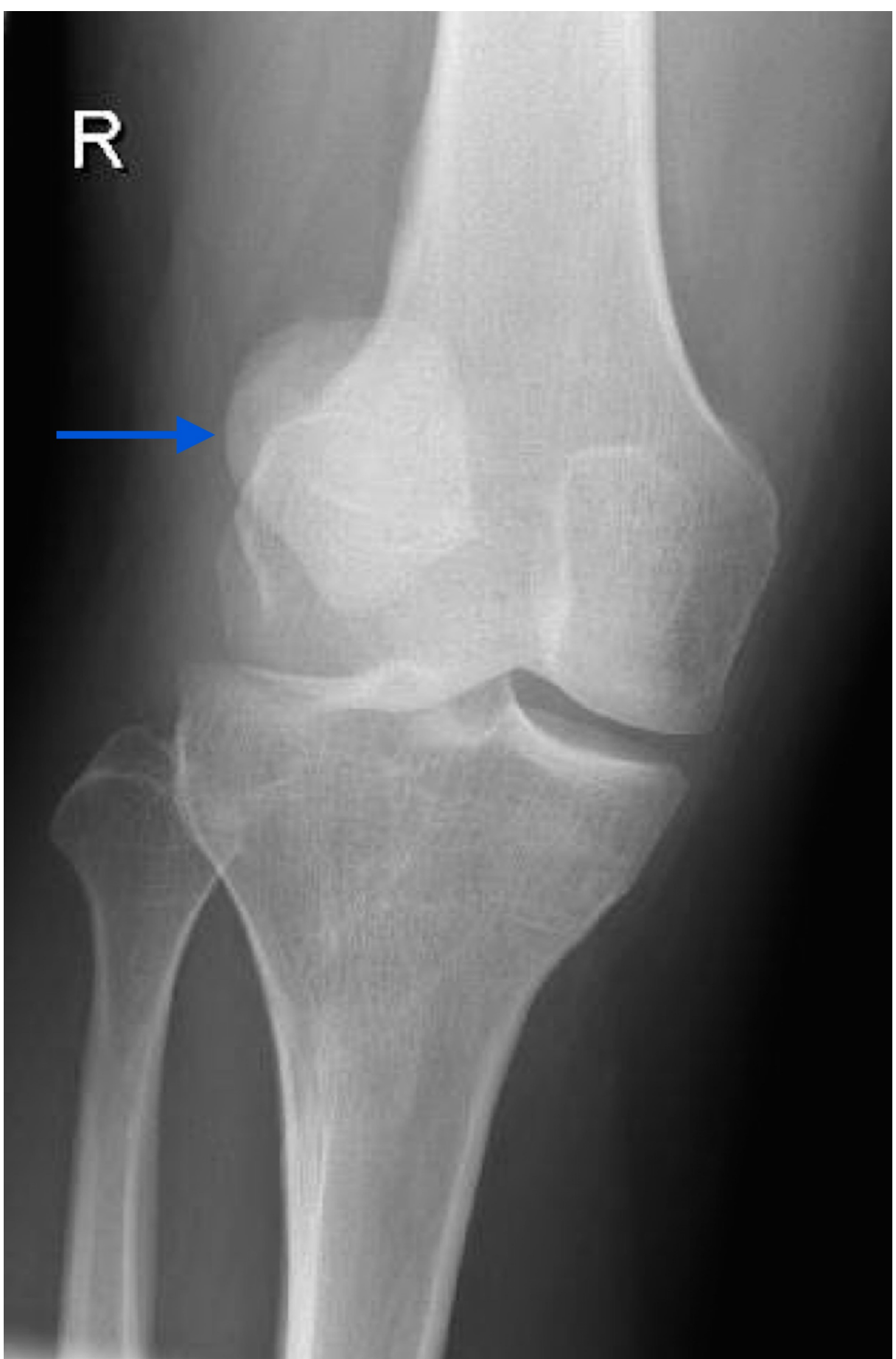

FIGURE 1: Plain anteroposterior radiograph of right knee showing lateral patellar displacement (blue arrow). 


\section{Cureus}

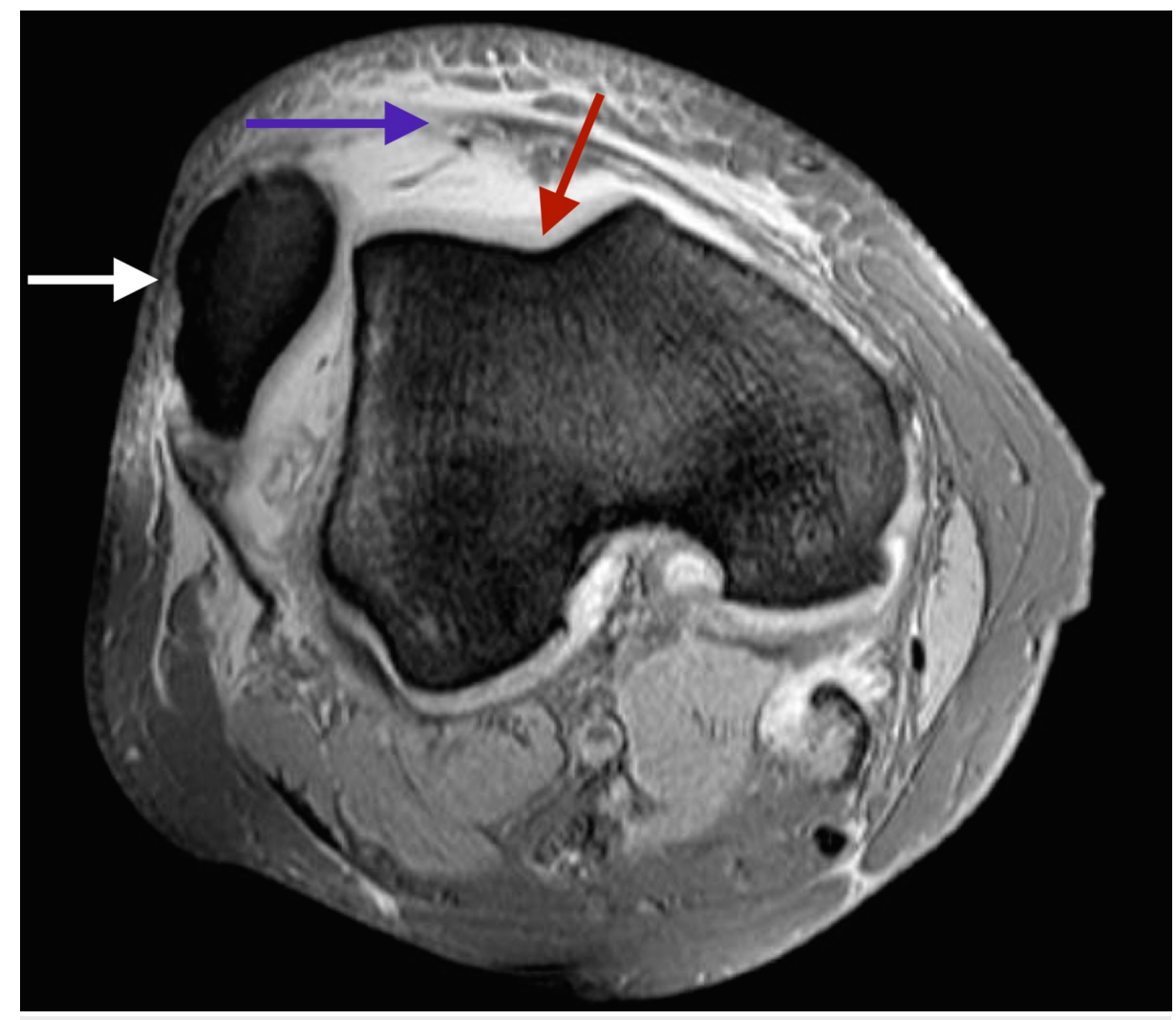

FIGURE 2: Axial magnetic resonance image of right knee showing lateral patellar dislocation (white arrow), bony avulsion of medial patellofemoral ligament (purple arrow) and a raised sulcus angle (red arrow). 


\section{Cureus}

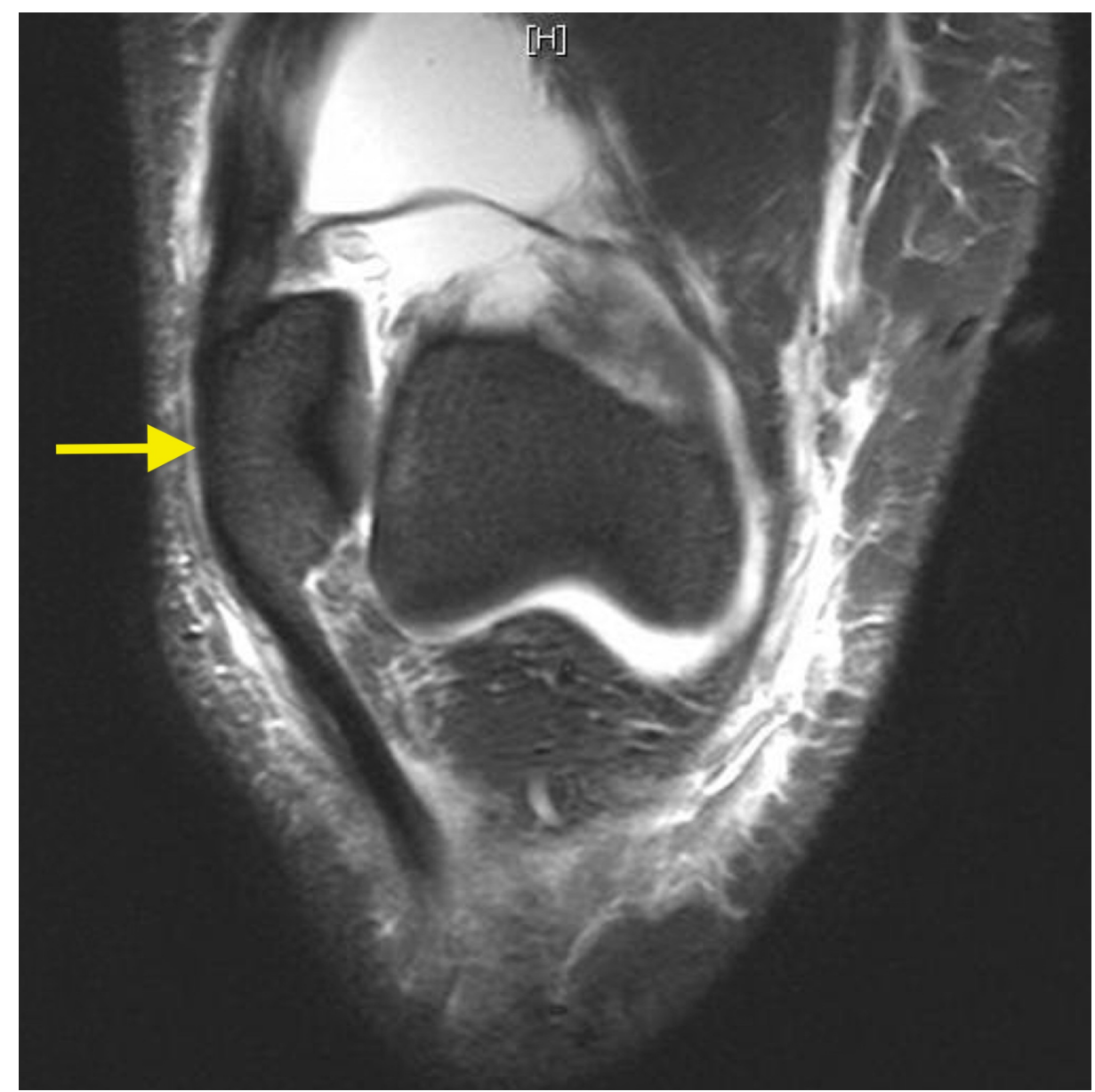

FIGURE 3: Lateralised, dislocated patella (yellow arrow) on coronal magnetic resonance image of right knee. 


\section{Cureus}

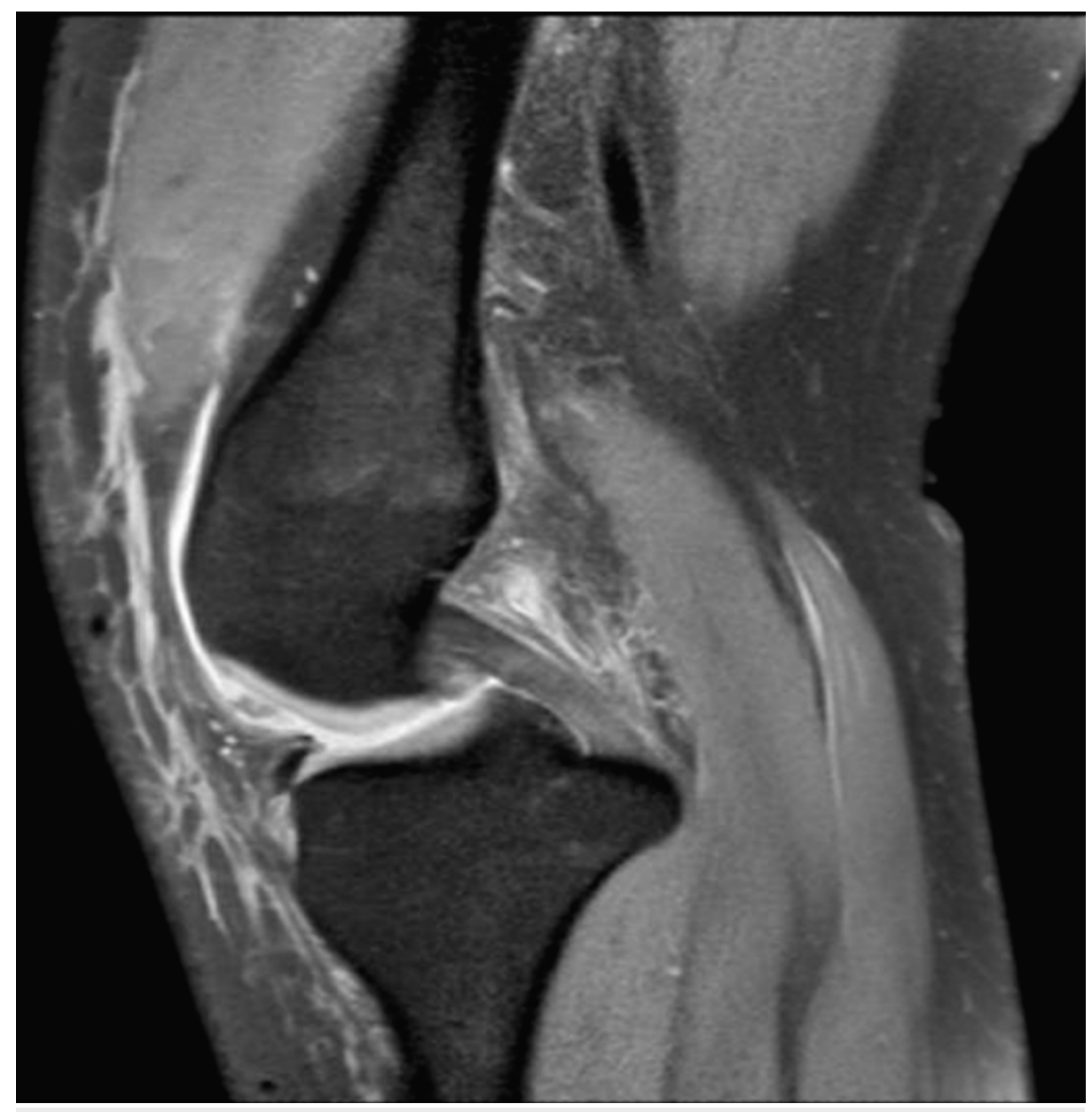

FIGURE 4: Sagittal magnetic resonance image of right knee.

In the context of failed closed reduction as well as confirmation of the diagnosis, associated soft-tissue injuries and presence of risk factors for recurrent dislocation, this patient subsequently proceeded to definitive surgical treatment in the form of MPFL repair, with excellent postoperative function and no further dislocation at 46 months postoperatively.

\section{Discussion}

Acute patellar dislocations are debilitating, with up to $58 \%$ of patients noting persistent limitation to strenuous activity and $55 \%$ failing to return to sport at six months following index injury [3,4]. While the majority of patients do not suffer from recurrent dislocation, those suffering from recurrence are at higher risk of persistent symptoms as well as patellofemoral osteoarthritis [2,3,5]. As a result, management of acute patellar dislocations in an attempt to address the risk of recurrence in the acute setting remains under debate, with a recent increase in acute surgical management occurring with potentially more favourable functional status, recurrence and complication rates but conservative management continuing to show acceptable long-term outcomes $[3,6]$.

Detailed anatomical illustration of the patellofemoral joint and surrounding structures at time of injury has thus become increasingly important aspect of initial evaluation following this injury [6-8]. This is typically achieved through MRI, which has an increasingly prominent role in helping dictate the definitive decision on nonoperative versus operative treatment in acute patellar dislocations [2]. MRI is particularly useful in detecting diagnostic factors of patellar dislocation, namely joint effusion, cartilaginous contusions of the lateral femoral condyle and medial patella, the presence of osteochondral lesions as well as the characteristically associated MPFL and medial retinaculum tears [1]. Additionally, it allows for surveillance of concomitant knee pathology. While a patient specific judgement, the presence of osteochondral injury as well as the existence of risk factors for recurrent dislocation, which include MPFL injury, raised sulcus angle, trochlear dysplasia, raised tibial tuberosity-trochlear groove distance, and an increased Insall-Salvati Index, pathognomonic of patella alta, each of which is assessed on MRI with high levels of specificity may indicate that surgical intervention is warranted $[1-3,9]$. 
Given both the increased diagnostic role of MRI and the relative frequency in which this injury is encountered, particularly in the adult population, awareness of both the plain radiographic features and more importantly the MRI findings associated with acute traumatic patellar dislocation as discussed above may aid in improved detection, earlier management and prevention of recurrent dislocations, hence reducing the potential debilitating effect of this injury $[1,2]$.

\section{Conclusions}

Traumatic patellar dislocations most commonly occur laterally and are associated with injury to both the medial patellofemoral ligament and medial patellar retinaculum. MRI is becoming an increasingly important diagnostic tool in assessment of traumatic patellar dislocations, useful in both detecting associated cartilage and soft tissue disruption as well as risk factors for recurrent dislocation. As such, awareness of the typical MRI findings in traumatic patellar dislocations outlined in the above case may potentially aid in pursuing either definitive conservative or surgical treatment in this pathology.

\section{Additional Information}

\section{Disclosures}

Human subjects: Consent was obtained by all participants in this study. Conflicts of interest: In compliance with the ICMJE uniform disclosure form, all authors declare the following: Payment/services info: All authors have declared that no financial support was received from any organization for the submitted work. Financial relationships: All authors have declared that they have no financial relationships at present or within the previous three years with any organizations that might have an interest in the submitted work. Other relationships: All authors have declared that there are no other relationships or activities that could appear to have influenced the submitted work.

\section{References}

1. Petri M, Ettinger M, Stuebig T, Brand S, Krettek C, Jagodzinski M, Omar M: Current concepts for patellar dislocation. Arch Trauma Res. 2015, 4:29301. 10.5812/atr.29301

2. Tsai C-H, Hsu C-J, Hung C-H, Hsu H-C: Primary traumatic patellar dislocation. J Orthop Surg Res. 2012, 7:21. 10.1186/1749-799X-7-21

3. Jain NP, Khan N, Fithian DC: A treatment algorithm for primary patellar dislocations . Sports Health. 2011, 3:170-174. 10.1177/1941738111399237

4. Atkin DM, Fithian DC, Marangi KS, Stone ML, Dobson BE, Mendelsohn C: Characteristics of patients with primary acute lateral patellar dislocation and their recovery within the first 6 months of injury. Am J Sports Med. 2000, 28:472-479. 10.1177/03635465000280040601

5. Lim AK, Chang HC, Hui JH: Recurrent patellar dislocation: reappraising our approach to surgery . Ann Acad Med Singapore. 2008, 37:320-323.

6. Longo UG, Ciuffreda M, Locher J, Berton A, Salvatore G, Denaro V: Treatment of primary acute patellar dislocation: systematic review and quantitative synthesis of the literature. Clin J Sport Med. 2017, 27:511523. 10.1097/jsm.0000000000000410

7. Koh JL, Stewart C: Patellar instability. Orthop Clin North Am. 2015, 46:147-157. 10.1016/j.ocl.2014.09.011

8. Smith TO, Donell S, Song F, Hing CB: Surgical versus non-surgical interventions for treating patellar dislocation. Cochrane Database Syst Rev. 2011, 008106. 10.1002/14651858.CD008106.pub2

9. Stefancin JJ, Parker RD: First-time traumatic patellar dislocation: a systematic review . Clin Orthop Relat Res. 2007, 455:93-101. 10.1097/BLO.0b013e31802eb40a 